\title{
READOUT ASIC FOR 3D POSITION-SENSITIVE DETECTORS ${ }^{*}$
}

\author{
Gianluigi De Geronimo, Emerson Vernon, Kim Ackley, \\ Angelo Dragone, Jack Fried, and Paul O'Connor \\ Brookhaven National Laboratory \\ Upton, NY 11973-5000 \\ Zhong He, Cedric Herman, and Feng Zhang \\ Department of Nuclear Engineering and Radiological Sciences \\ University of Michigan, Ann Arbor, MI
}

October, 2007

\footnotetext{
*This manuscript has been authored by Brookhaven Science Associates, LLC under Contract No. DE-AC02-98CH10886 with the U.S. Department of Energy. The United States Government retains, and the publisher, by accepting the article for publication, acknowledges, a world-wide license to publish or reproduce the published form of this manuscript, or allow others to do so, for the United States Government purposes.
} 


\section{DISCLAIMER}

This work was prepared as an account of work sponsored by an agency of the United States Government. Neither the United States Government nor any agency thereof, nor any of their employees, nor any of their contractors, subcontractors, or their employees, makes any warranty, express or implied, or assumes any legal liability or responsibility for the accuracy, completeness, or any third party's use or the results of such use of any information, apparatus, product, or process disclosed, or represents that its use would not infringe privately owned rights. Reference herein to any specific commercial product, process, or service by trade name, trademark, manufacturer, or otherwise, does not necessarily constitute or imply its endorsement, recommendation, or favoring by the United States Government or any agency thereof or its contractors or subcontractors. The views and opinions of authors expressed herein do not necessarily state or reflect those of the United States Government or any agency thereof. 


\title{
Readout ASIC for 3D Position-Sensitive Detectors
}

\author{
Gianluigi De Geronimo, Emerson Vernon, Kim Ackley, Angelo Dragone, Jack Fried, Paul O'Connor \\ Zhong He, Cedric Herman, and Feng Zhang
}

\begin{abstract}
We describe an application specific integrated circuit (ASIC) for 3D position-sensitive detectors. It was optimized for pixelated CZT sensors, and it measures, corresponding to an ionizing event, the energy and timing of signals from 121 anodes and one cathode. Each channel provides low-noise charge amplification, high-order shaping, along with peak- and timing-detection. The cathode's timing can be measured in three different ways: the first is based on multiple thresholds on the charge amplifier's voltage output; the second uses the threshold crossing of a fast-shaped signal; and the third measures the peak amplitude and timing from a bipolar shaper. With its power of $2 \mathrm{~mW}$ per channel the ASIC measures, on a CZT sensor connected and biased, charges up to $100 \mathrm{fC}$ with an electronic resolution better than $200 \mathrm{e}^{-} \mathrm{rms}$. Our preliminary spectral measurements applying a simple cathode/anode ratio correction demonstrated a single-pixel resolution of $4.8 \mathrm{keV}(0.72 \%)$ at $662 \mathrm{keV}$, with the electronics and leakage current contributing in total with $2.1 \mathrm{keV}$.
\end{abstract}

\section{INTRODUCTION}

Cadmium zinc telluride (CZT) sensors have emerged as leading candidates in room-temperature, large-volume gamma-ray spectrometry for security-, medical-, industrial-, and spaceapplications [1-7]. More recently another wide-bandgap material, mercuric iodide $\left(\mathrm{HgI}_{2}\right)$, was suggested as potential candidate [8-10]. Both materials can be produced with relatively low dark current, but their spectral resolution is strongly limited by several deficiencies, viz., the poor mobility of holes, electron trapping, and extensive non-uniformities. Accordingly, various solutions were proposed that link suitable electrode configurations with bi-parametric signal correction [11-19].

The 3-D Position-Sensitive Detector (3DPSD) [20-22] combines the pixelation of the anode electrode with the measurement of amplitude and timing. This information is used to reconstruct the position of the ionizing interaction, and to correct the measurement on a voxel-by-voxel basis (voxel stands for volumetric pixel), thus compensating for the deficiencies. With this approach, were registered energy resolutions better than $1 \%$ FWHM at $662 \mathrm{keV}$ in CZT, limited only by the resolution of the readout electronics. Additionally, employing the 3D correction method allows the use of lower-grade and larger size detectors in a larger number of applications.

We developed an application specific integrated circuit (ASIC) for 3D position-sensitive detectors. The ASIC provides low-noise charge amplification, filtering, and measurements of amplitude and timing on signals from the cathode and 121 anodes.

In Section II, we introduce the architecture of the ASIC, and discuss some new circuit solutions. Section III describes meth-

G. De Geronimo (degeronimo(abnl.gov), E. Vernon, K. Ackley, J. Fried, and P. O'Connor are with the Instrumentation Division, Brookhaven National Laboratory, Upton, NY.

A. Dragone is with the National Synchrotron Light Source, Brookhaven National Laboratory, Upton, NY.

Z. He, C. Herman, and F. Zhang are with the Department of Nuclear Engineering and Radiological Sciences, University of Michigan, Ann Arbor, MI. ods for the timing measurements, wherein an innovative approach consists of applying a bipolar shaping to the cathode signal. Section IV reports our first experimental results.

\section{ARCHITECTURE OF THE ASIC}

The ASIC was designed to read-out the signals from pixelated CZT sensors similar to that in Fig. 1(a), characterized by $11 \times 11(121)$ anodes and one cathode. In correspondence to an ionizing event, the ASIC measures the peak amplitude and relative timing from each of these 122 electrodes. It also provides an analog charge-amplifier monitor for the cathode and for the anode-grid signals. Reading out the anode signals entails negative-charge amplification, while that from the cathode and anode-grid requires the opposite.

The ASIC is wire-bonded to a $22 \times 22 \mathrm{~mm}^{2}$ interposer (Fig. 1) that includes on one side three sockets (Fig. 1(b)), for the CZT sensor's connection, and, on the other side, two sockets (Fig. 1(c)), for the analog- and digital-supplies and signals. The ASIC inputs are symmetrically located along its left and right sides, the analog outputs and supplies are at the bottom, while the digital signals and supplies are at the top. The three large holes at the bottom of the interposer are for the connection of the cathode signal and for the bias of the anode-grid $(\approx 100 \mathrm{~V})$. The cathode's bias circuitry, not shown, is placed close to the cathode electrode. The interposer is connected to a mother board that assures a regulated supply and can accommodate nine interposers in a $3 \times 3$ configuration.
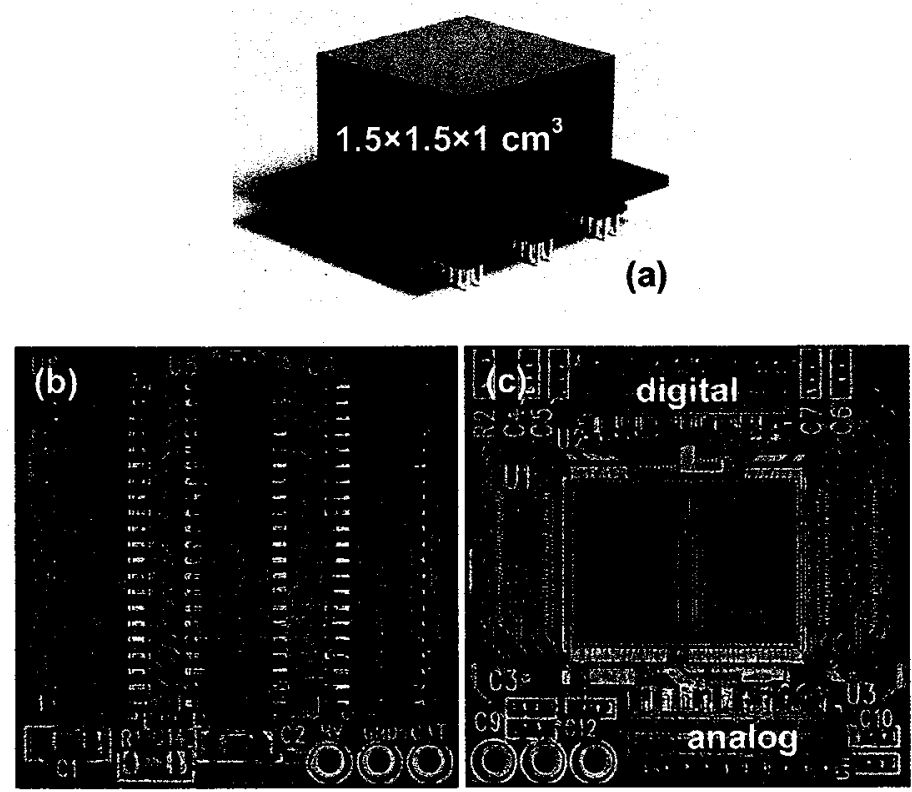

Fig. 1 - Sample of a 121 anodes pixelated CZT sensor (a); interposer front (ASIC) view (a), and back (sensor) view (c).

Fig. 2 is a block diagram of the ASIC that comprises 124 channels (122 read-out negative charge, two read-out positive), 
bias circuitry, six 10-bit DACs, two test-signal generators, one temperature sensor, 1010-bit configuration register, logic for configuration and readout, multiplexers, and analog monitors.

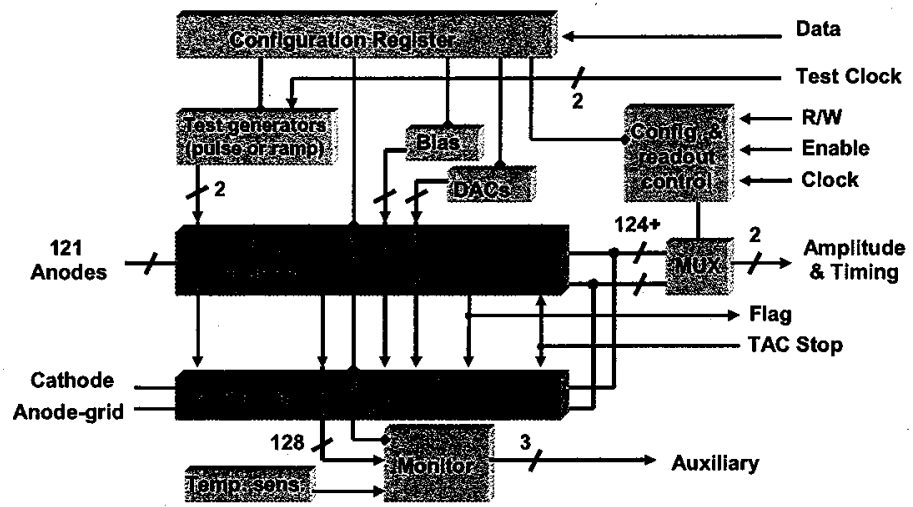

Fig. 2 - Block diagram of the ASIC.

Each of the 122 anode channels, shown in Fig. 3, implements a low-noise charge amplifier with single-stage-compensated adaptive continuous reset [23] having charge gain of 48. The input MOSFET is a p-channel device optimized for $3 \mathrm{pF}$ input capacitance [24], with gate size $\mathrm{L} / \mathrm{W}=0.36 \mu \mathrm{m} / 300 \mu \mathrm{m}$ and biased at a drain current of about $100 \mu \mathrm{A}$, corresponding to 250 $\mu \mathrm{W}$ dissipated in the input branch. With a transconductance $\mathrm{g}_{\mathrm{m}}$ $\approx 1.7 \mathrm{mS}$ and a gate capacitance $\mathrm{C}_{\mathrm{g}} \approx 650 \mathrm{fF}$ the input MOSFET contributes to the ENC with about 75 electrons rms at $3 \mathrm{pF}$ input capacitance (assuming a high order shaper with $1 \mu$ s peaking time).

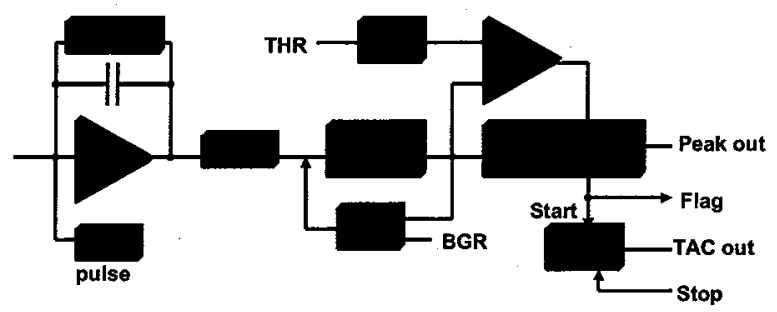

Fig. 3 - Block diagram of the anode channel.

The channel implements some circuit solutions for voltage amplifiers adopted in the charge amplifier and shaper stages. These solutions improved performance while maintaining the power dissipated in the channel within specifications.

The input MOSFET $M_{1}$ is followed by a dual-cascode stage $\mathrm{M}_{\mathrm{Cl}}, \mathrm{M}_{\mathrm{C} 2}$, that increases the amplifier's dc gain and lowers the impedance at the drain node of $M_{1}$. This solution improves the amplifier's performance without increasing the dissipated power. The voltage drop across $\mathrm{M}_{\mathrm{C} 1}$ has negligible impact in our charge amplifier's configurations based on continuous reset. The typical gate width of $M_{C l}$ is about one third of $M_{1}$. We give details about this solution elsewhere [25].

For voltage amplifiers that must provide negative swing at the output, the capacitor $C_{A}$ was introduced. For negative output swings, the voltage at the gain node (drain node of the load $\mathrm{M}_{\mathrm{L}}$ ) is slew-rated, limited by the current in $\mathrm{M}_{\mathrm{L}}$. When this occurs, the input voltage of the amplifier suddenly increases, and the drain voltage of the source $M_{S}$ falls. The capacitor $C_{A}$ uses this decrease to send additional current to the gain node, thereby improving the performance for large signals without increasing dissipated power. A typical value of $\mathrm{C}_{\mathrm{A}}$ is about $1 \mathrm{pF}$.

The output stage consists of a source follower configuration $\mathrm{M}_{\mathrm{F}}, \mathrm{M}_{\mathrm{FS}}$ assisted by its scaled-down replica $\mathrm{M}_{\mathrm{Fa}}, \mathrm{M}_{\mathrm{FSa}}$ and by a differential amplifier, generating a local feedback. The dc gate voltage of the source $M_{F S}$ is forced to the gate voltage of $M_{S a}$. During transients, the gate voltage of $\mathrm{M}_{\mathrm{FS}}$ is dynamically adjusted, increasing or decreasing the current so that the loaded output voltage follows its unloaded replica. This solution allows a reduction in the bias current in the follower, while maintaining the required performance. The power dissipated by the replica and the differential amplifier can be contained within negligible values. The network $\mathrm{R}_{\mathrm{FC}}, \mathrm{C}_{\mathrm{FC}}$, with typical values of 10 $\mathrm{k} \Omega, 100 \mathrm{fF}$, provides compensation and bypasses the active loop at the highest frequencies. Typical currents through the $\mathrm{M}_{\mathrm{FS}}, \mathrm{M}_{\mathrm{FSa}}$, and differential stage are, respectively, about $20 \mu \mathrm{A}$, $1 \mu \mathrm{A}$, and $4 \mu \mathrm{A}$. .

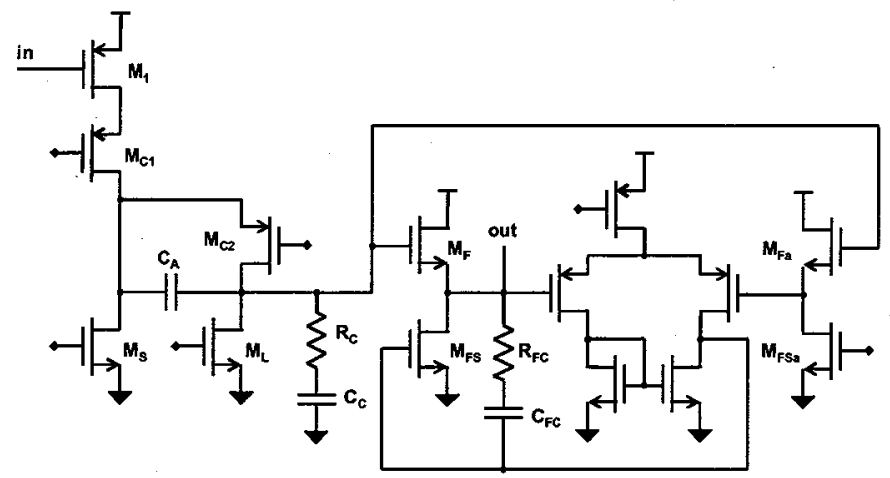

Fig. 4 - Schematic of the voltage amplifier that implements some circuit solutions.

The charge amplifier is followed by a $5^{\text {th }}$ order shaper with complex conjugate poles, an adjustable peaking time (0.25-, $0.5-, 1-$, and 2- $\mu \mathrm{s})$, and an output baseline stabilized with a Baseline Holder (BLH) [26]. It follows a discriminator with 4bit trimmer, a peak and timing detector, and a Time-toAmplitude Converter (TAC) (the duration of ramp is adjustable to 1-, 2-, 3-, and 4- $\mu \mathrm{s}$ ). The amplitude of the pulse is measured and stored in an analog memory using a multi-phase peakdetection configuration [27]. The TAC converts the timing of the anode event into a voltage. . The ramp starts at a trigger released by the peak detector at the time of the peak, and is stopped by an external trigger [28]. Section III has details of this timing method. The channel includes a mask function and a test capacitor connected to a test-pulse generator, common to all channels, whose amplitude is controlled by a 10-bit DAC. The shaped pulse from it can be routed to an auxiliary analog output (analog monitor). The entire channel is characterized by a gain of about $20 \mathrm{mV} / \mathrm{fC}$ (about $18 \mathrm{mV} / \mathrm{fC}$ at $0.25 \mu$ s peaking time) and dissipates about $1.35 \mathrm{~mW}$.

Fig. 5 shows the two channels for the cathode and anodegrid, each of which implements a low-noise charge amplifier with dual stage adaptive reset having total charge gain of 48 . 
The input MOSFET is a p-channel device optimized for $6 \mathrm{pF}$ input capacitance, with gate size $\mathrm{L} / \mathrm{W}=0.36 \mu \mathrm{m} / 600 \mu \mathrm{m}$ and biased at a drain current of about $200 \mu \mathrm{A}$, corresponding to 500 $\mu \mathrm{W}$ dissipated in the input branch. With $\mathrm{a}_{\mathrm{m}} \approx 3.4 \mathrm{mS}$ and a $\mathrm{C}_{\mathrm{g}}$ $\approx 1.3 \mathrm{pF}$, it contributes to the ENC with about 100 electrons rms at $6 \mathrm{pF}$ input capacitance (assuming a high order shaper with 1 $\mu$ s peaking time). The remainder of the channel splits in three sections providing, respectively, energy measurement, timing measurement, and a preamplifier monitor (the latter not shown in Fig. 5).

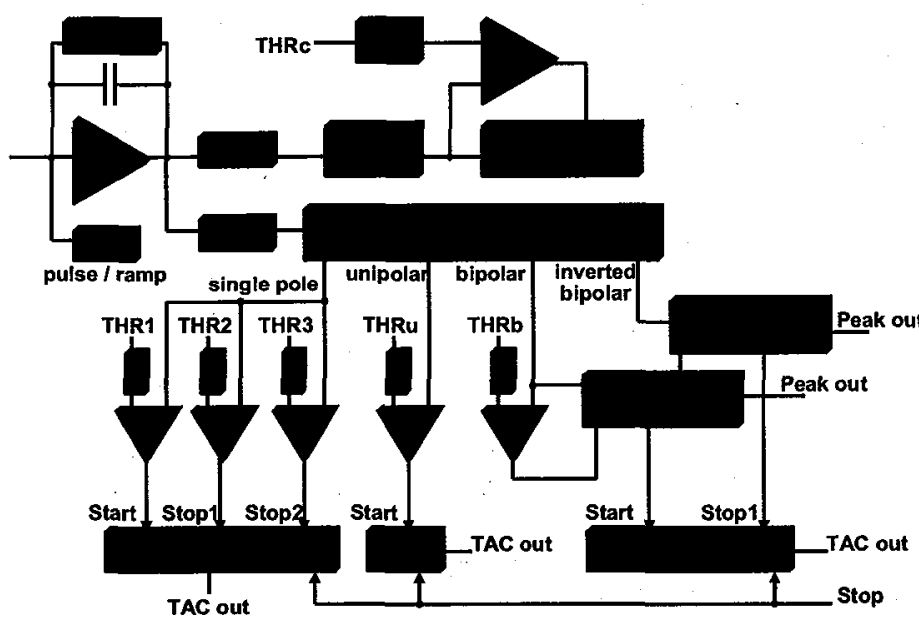

Fig. 5 - Block diagram of the cathode channel.

The cathode's energy section implements a $5^{\text {th }}$ order shaper with complex conjugate poles and an adjustable peaking time (0.25-, 0.5-, 1-, and 2- $\mu \mathrm{s})$, a BLH, a discriminator with 4-bit trimmer, and a multi-phase peak detector. The cathode's timing section includes a multi-stage shaper with four outputs: the first provides a replica of the charge preamplifier's output, the second a $3^{\text {rd }}$ order unipolar-shaping with complex conjugate poles and an adjustable peaking time (100-, 200-, 400-, and 800-ns), while the third provides a differentiation on the unipolar shaping, meaning a bipolar shaping (peaking time about 50-, 100-, $200-$, and $400 \mathrm{~ns}$ ). The fourth output constitutes an inversion of the third output. These four outputs measure the timing of the cathode's signals via three different timing methods, discussed in detail in Section III. The timing circuits use discriminators with 4-bit trimmers, peak detectors and TACs (ramp durations adjustable to 1-, 2-, 3-, and 4- $\mu \mathrm{s})$. The channel has a mask function and a test capacitor connected to a pulse or ramp generator, common to all channels, the amplitude of which is controlled by a 10-bit DAC. Test ramps can be generated with adjustable slope and duration, thus simulating actual cathode signals. For monitoring, the charge amplifier's voltage output is available through a dedicated output (preamplifier monitor). The shaped pulse from the energy and timing sections can be routed to the auxiliary analog output (analog monitor). The whole channel is characterized by a gain of about $20 \mathrm{mV} / \mathrm{fC}$ for the energy section, and about 23 and $15 \mathrm{mV} / \mathrm{fC}$, respectively, for the unipolar shaper and bipolar shaper in the timing section; it dissipates overall about $5.25 \mathrm{~mW}$.
The ASIC can operate either in an acquisition or readout mode, controlled by an external LVDS EN. In the former, entered by setting EN on high, all channels become sensitive to input events. When an event occurs and a first peak is found, the ASIC releases a LVDS flag, FL, and then the external electronics can stop acquisition by lowering the EN. This signal also is used as stop trigger for the TACs. Having entered the readout mode, all analog outputs (energy and timing) from all channels are made sequentially available, with a LVDS clock CK, at two dedicated analog outputs.

The ASIC discriminates events based only on anode signals. Once this is enabled (with EN high), should an anode signal exceed the threshold, then the peak amplitude and timing measurements are enabled in all other anodes. The flag FL is released, after a delay of $200 \mathrm{~ns}$, at the time the first peak is found. The anodes process the amplitude and timing of the highest peak until the acquisition stops (EN low). In one option, the timing can be measured only from the first peak; this choice option can be valuable for extracting timing information from inductions occurring in those pixels neighboring the ones above threshold. In another option, channels that did not exceed the threshold can be forced to store that amplitude at the time of the first above-threshold peak. This one can be useful to force the sampling of negative amplitudes that may result from induction in the pixels next to the ones above threshold.

A cathode signal exceeding the threshold is always processed for peak amplitude and timing, but the event will not trigger the flag FL. The cathode will continue processing the amplitude and timing of the highest peak until the acquisition stops (EN low).

Except for the serial configuration signals, all the digital inputs and outputs are Low Voltage Differential Signals (LVDS). The ASIC includes one test-pulse generator for the anodes and one test pulse/ramp generator for the cathode, controlled by independent LVDS clock signals and two 10-bit DACs. The common thresholds are controlled by four 10-bit DACs and a one 4-bit DAC. The temperature sensor is characterized by a nominal response of $1.52 \mathrm{~V}+5.67 \mathrm{mV} /{ }^{\circ} \mathrm{C}$. The outputs from the DACs and temperature sensor can be routed to the auxiliary analog output (analog monitor). The whole ASIC dissipates 250 $\mathrm{mW}$, with an effective power per channel of about $2 \mathrm{~mW}$. Constructed in CMOS $0.25 \mu \mathrm{m}$, the die, measuring $11.3 \mathrm{~mm} \times 8.2$ $\mathrm{mm}$, has 208 input/output pads.

\section{TIMING Methods}

An ionizing event produces electron-hole pairs in CZT material in an amount proportional to the energy of the event. Under the applied voltage, the electrons and holes move, respectively, from the point of interaction to the anodes and cathode. . The currents induced in both are measured by the associated charge amplifier. Because the holes have poor transport properties, only the electrons effectively contribute to the currents.

The signal induced in the cathode, a planar electrode, starts at the time of interaction and stops when the electrons reach the anodes, to which the electrons travel at a speed that depends 
upon the applied voltage. The duration of the induced signal depends on the applied voltage and depth of interaction: it is longer when the interaction is in the proximity of the cathode (since the electrons travel through the whole sensor), and declines to negligible values as the interaction occurs closer to the anodes. Consequently, for a given energy, the total induced charge is higher when the interaction occurs close to the cathode and decreases to negligible when the interaction is closer to the anodes. The voltage signal at the output of the charge amplifier, which is the integral of the induced current, is, to a firstorder, a ramp with a slope proportional to the energy (i.e., to the total charge generated by the ionization) and duration proportional to the depth of interaction. For a given energy, the final amplitude of the ramp is proportional to the depth of interaction.

The signal induced in the anode is subject to the small-pixel effect $[29,30]$ that remains negligible (since it is distributed among all anodes and anodes-grid) until the electrons approach the anodes at a distance of about one pixel pitch. Then a strong current is induced in those anodes that collect the charge. Differently from the cathode, the total induced charge is, , to a first order, independent of the depth of interaction and only depends on the event's energy. The voltage signal at the output of the charge amplifier is, to a first-order, a step with amplitude proportional to the energy, independent of the depth of the interaction. Compared to planar electrodes, the small-pixel configuration effectively makes the detector sensitive to only one kind of charges, the electrons.

In the absence of electron trapping, and assuming an ideal small-pixel effect, the total charge measured at the anode (or anodes) is proportional to the event's energy. Taking electron trapping and second-order small-pixel effects into account, then the anode signals show a non-negligible dependence on the depth of interaction that considerably affects spectral performance.

This dependence can be compensated for by measuring, for each event, both the amplitude of the signal at the anode and the depth of the interaction, and by applying to the amplitude the associated corrective coefficient. The depth of interaction can be extracted to a first order from the ratio between the amplitude of the cathode signal and the one of the anode (or sum of anodes). This approach is effective for ionizing events having a single point of interaction or involving only a few anodes. For events with multiple points of interaction or encompassing several anodes, the depth of each point can be more efficiently extracted from measuring the relative drift time associated with each anode signal, i.e,. the delay from the time of interaction, measured on the cathode signal, to the time the electrons reach each anode. All these bi-parametric approaches afford correction coefficients for each volumetric pixel (voxel) in the sensor [20-22,30].

Techniques were implemented in the ASIC to accurately measure the timing of the anode and cathode signals.

The timing of the former is obtained, as anticipated in the previous Section, by measuring the peaking time of the anodeshaped pulse. In contrast to timing measured from the threshold crossing on unipolar pulses, this peaking-time method has the advantage of being, to a first order, independent of the amplitude. In principle, the timing of the peak-found signal of the peak detector circuit [27], shown in Fig. 6, is equal to the one of the zero-crossings on a bipolar pulse. The current flowing through the hold capacitor $C_{h}$ during peak detection equals the derivative of the input pulse according to $i_{h}=C_{h} d V_{i} / d t$. When the signal $V_{i}$ approaches the peak, the current $i_{h}$ approaches zero, and the voltage, $\mathrm{V}_{\mathrm{g}}$ equals $\mathrm{V}_{\mathrm{DD}}-\mathrm{V}_{\mathrm{THh}}$. Immediately after the peak, the signal, $V_{i}$, starts falling and, due to the high gain, $G_{d}$, of the differential amplifier the output voltage, $V_{g}$, sharply increases towards $V_{\mathrm{DD}}$ crossing the comparator threshold, $\mathrm{V}_{\mathrm{TH}}$, set at a voltage $V_{D D}-V_{T H h}<V_{T H}<V_{D D}$, where $V_{T H h}$ is the threshold voltage of the controlled current source $M_{h}$.

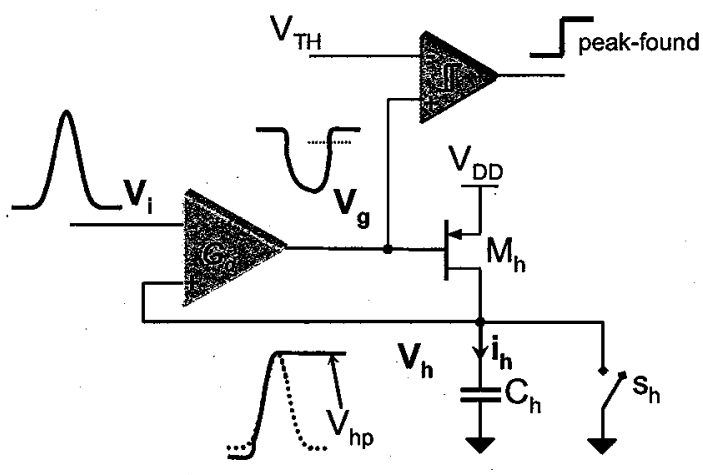

Fig. 6 - Block diagram of the peak detector's circuit.

The timing resolution $\sigma_{t}$ achievable with this method can be approximated with [31]

$$
\sigma_{t} \approx \frac{\sigma_{i h}}{i_{h}^{\prime}\left(\tau_{p 0}\right)} \approx \frac{i_{h p}}{Q} \frac{\operatorname{ENC}_{b}\left(\tau_{p b}\right)}{i_{h}^{\prime}\left(\tau_{T H}\right)} \approx \frac{\operatorname{ENC}_{b}\left(\tau_{p} \eta_{p}\right) \tau_{p} \lambda_{p}}{Q \rho_{p}},
$$

where $\sigma_{\text {ih }}$ is the noise on the current signal $\dot{i}_{h}$, and $\dot{i}_{h}{ }^{\prime}$ is the derivative calculated at the time, $\tau_{\mathrm{TH}}$, of the threshold crossing of the comparator, where $\mathrm{i}_{\mathrm{h}}\left(\tau_{\mathrm{TH}}\right)=\mathrm{i}_{\mathrm{hTH}}$ then is the current. We note that $i_{\mathrm{hTH}}$ is set by the threshold $\mathrm{V}_{\mathrm{TH}}$, and, for $\tau_{\mathrm{TH}}$ approaching the time $\tau_{\mathrm{p} 0}$ at which $\mathrm{V}_{\mathrm{i}}$ peaks, it follows that $\mathrm{i}_{\mathrm{h}}{ }^{\prime}\left(\tau_{\mathrm{TH}}\right) \approx \mathrm{i}_{\mathrm{h}}{ }^{\prime}\left(\tau_{\mathrm{p} 0}\right)$. In (1), the noise $\sigma_{\text {ih }}$ is assumed to be dominated by that on the shaped pulse, $\mathrm{V}_{\mathrm{i}}$, from the first front-end stage, and it is calculated in (1) as $\mathrm{ENC}_{\mathrm{b}}$ multiplied by the gain from the signal charge $Q$ to the current $i_{h}$ in $C_{h}$ (i.e., $i_{h p} / Q$, where $i_{h p}$ is the peak value of $i_{h}$ ). Therefore, the term $E C_{b}$ is calculated assuming a bipolar shape (current $i_{h}$ ) with peaking time $\tau_{p b}$. Normalized coefficients can be adopted [31], yielding the final expression in (1) that depends only on $Q$ and the peaking time $\tau_{p}$ of $V_{i}$. For a $5^{\text {th }}$ order shaper with complex conjugate poles, they are $\rho_{p}=$ $3.65, \eta_{p}=0.58$, and $\lambda_{p}=1.58$ [31]. Assuming a unipolar pulse with peaking time $1 \mu \mathrm{s}$ and resolution $200 \mathrm{e}^{-} \mathrm{rms}$, the timing. resolution $\sigma_{t}$ from (1) can be plotted as done in Fig. 7. In the same figure, the resolution for threshold crossing on the same unipolar pulse is depicted, along with the associated time walk, assuming a threshold at $0.5 \%$ of the maximum amplitude. 


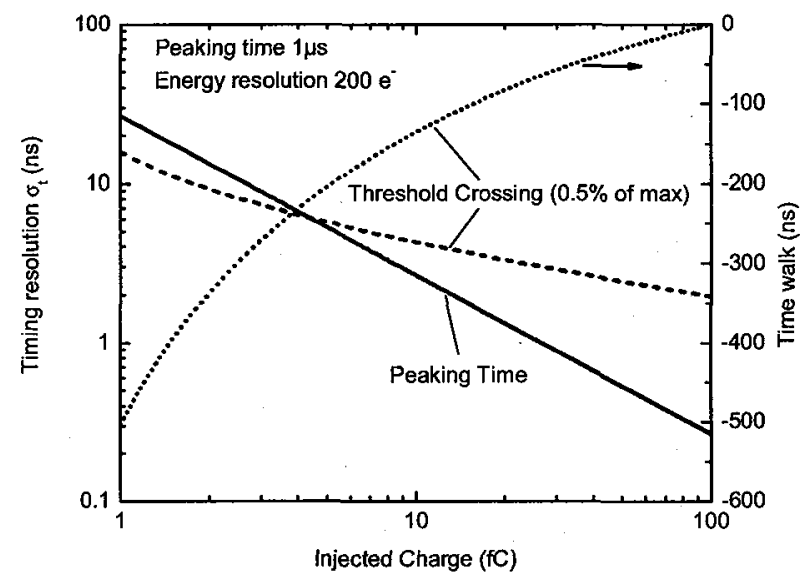

Fig. 7 - Theoretical timing resolution from (1) assuming a unipolar shape with peaking time of $1 \mu \mathrm{s}$ and energy resolution 300 electrons rms. The case of threshold crossing, along with the associated time walk, also is shown.

The results in (1) and Fig. 7 assume there is a negligible contribution to the noise from the shaper, the peak detector, the comparator, and the downstream stages. In practice, overlooking these other contributions (as we partially did) can severely limit the timing resolution. Particular attention must be paid to the residual high-frequency noise components from the last stages of the shaper, and from the differential amplifier of the peak detector. The signal, $i_{h}$, decreases with the peaking time, and its slope $\mathrm{i}_{\mathrm{h}}{ }^{\prime}\left(\tau_{\mathrm{p} 0}\right)$ declines with the square of the peaking time. On the other hand, these noise components, along with their emphasis by the derivative, are, to a first order, independent of peaking time. The contribution to the timing resolution then is proportional to the square of the peaking time, and can be approximated with $\sigma_{\text {ih_h }} / i_{\mathrm{h}}{ }^{\prime}\left(\tau_{\mathrm{p} 0}\right)$, where $\sigma_{\text {ih_hf }}$ is the corresponding rms current noise. A resistor placed in series with the capacitor $\mathrm{C}_{\mathrm{h}}$, to help stabilize the peak detector loop, also would filter part of such high-frequency noise. The noise from the comparator can be evaluated as $\sigma_{\mathrm{vd}} / \psi_{\mathrm{vg}}$, where $\sigma_{\mathrm{vc}}$ is the rms voltage noise at its input, and $\psi_{\mathrm{Vg}}$ is the slope of $\mathrm{V}_{\mathrm{g}}$. The slope can be approximated from the Taylor series of the signal, $\mathrm{V}_{\mathrm{i}^{-}}$ $\mathrm{V}_{\mathrm{ip}}$, immediately after the peak, yielding $\psi_{\mathrm{Vg}} \approx \mathrm{A} \cdot \operatorname{sqrt}(2$ $\left.\mathrm{i}_{\mathrm{h}}{ }^{\prime}\left(\tau_{\mathrm{p} 0}\right) \mathrm{V}_{\mathrm{ip}} / \mathrm{C}_{\mathrm{h}}\right)$ where $\mathrm{A}$ is the difference voltage gain. Again, this contribution, usually small, is proportional to the square of the peaking time. Assuming that the timing signal is converted into a voltage using a TAC (as in our case), the series noise from the TAC and its readout can be approximated with $\operatorname{sqrt}\left(\mathrm{S}_{\mathrm{TAC}} \mathrm{T}_{\mathrm{TAC}}\right) /\left(\psi_{\mathrm{TAC}} \mathrm{C}_{\mathrm{TAC}}\right)$, where $\mathrm{S}_{\mathrm{iTAC}}$ is the spectral noise power density of the current flowing into a capacitor $\mathrm{C}_{\mathrm{TAC}}$ to generate the ramp, $\mathbf{T}_{\mathrm{TAC}}$ is the ramp's duration, and $\psi_{\mathrm{TAC}}$ is its slope. The contribution from the downstream stages (e.g., the buffer, ADC) can be approximated with $\sigma_{\text {vo }} / \psi_{\text {TAC }}$ where $\sigma_{\text {vro }}$ is their rms input voltage noise. .

For measuring the timing of the cathode signals, we established three different methods: preamplifier multi-threshold, unipolar threshold, and bipolar peaking time. All take into account the cathode signal's relatively long induction time.

The preamplifier multi-threshold method is performed directly on the output voltage of the charge amplifier (before the shaping). As discussed, the voltage signal at the output of the charge amplifier is, to a first order, a ramp whose slope is proportional to the energy, and duration to the depth of interaction. By measuring the timing of the crossing of three different thresholds [32] the slope of the signal (i.e., the energy) can be reconstructed, and also the timing at the departing point of the ramp (i.e., the time of interaction). This method still requires energy information from the anodes for those signals that cross only the first threshold. A drawback here is the poor noise filtering, limited to the one introduced by the parasitics in the charge amplifier. For slow signals, as in our case, the noise may easily dominate the signal.

The unipolar threshold method, frequently adopted by designers, consists of measuring the timing at the threshold crossing of a fast-shaped cathode signal. It necessitates having an additional fast shaper associated with the cathode channel. Since the time at the crossing of the threshold depends on the pulse's amplitude and induction time, this measurement exhibits considerable dependence on the energy and depth of interaction. Accordingly, it requires additional calibration based on the energy information from the anodes.

The bipolar peaking time method is based on measuring amplitude and peaking time of the cathode signal filtered by a fast bipolar shaper; hence, an additional fast shaper must be added $\mathrm{d}$ to the cathode channel. Bipolar-shaped signals exhibit a unipolar response each time the slope of the charge amplifier voltage signal (i.e., the ramp) changes. The amplitude of the resulting unipolar pulse is proportional to the change in slope, and the timing is related only to the time of the change in slope. If the peaking time is small compared to the induction time, the shaped signal responds with a positive unipolar pulse when the ramp departs from the baseline, and with a negative unipolar pulse at the end of the ramp (Fig. 8). The amplitude of the positive pulse is proportional to the slope, and it provides a firstorder measurement of the energy. The peaking time is associated with the time the slope changes providing, to a first order, a timing measurement independent of the energy and depth of interaction. For events with single point of interaction, the delay from the positive to the negative peak affords a first order value for the electron drift time (i.e., the depth of interaction). The ASIC measures amplitude and timing of both positive and negative pulses.

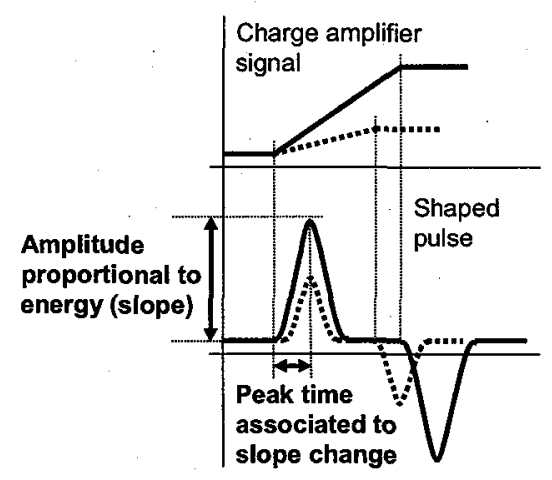

Fig. 8 - Diagram illustrating the bipolar peaking time method for measuring the timing of cathode signals. Two cases are illustrated, differing in energy and depth. . 
By directly measuring the drift time and energy without requiring the information from the anodes, the first and the last methods of cathode timing are expected to offer better overall performance. All three were designed to provide a timing resolution better than $20 \mathrm{~ns}$ for charges above $1 \mathrm{fC}$.

\section{FABRICATION AND FIRST EXPERIMENTAL RESULTS}

We fabricated the first prototype of the ASIC, with a layout size of $11.3 \mathrm{~mm} \times 8.2 \mathrm{~mm}$, in a commercial CMOS $0.25 \mu \mathrm{m}$ technology. Fig. 9 is a picture of the die. The 122 anode channels plus two cathode channels are symmetrically arranged with 62 inputs on the left, and 62 on the right. The bias and readout circuitry are in the center, the analog outputs at the bottom, and the digital interface at the top. The the channels are, respectively, $100 \mu \mathrm{m} \times 5 \mathrm{~mm}$ and $200 \mu \mathrm{m} \times 5 \mathrm{~mm}$ for the anode and cathode. The total transistor count is about 300,000 .

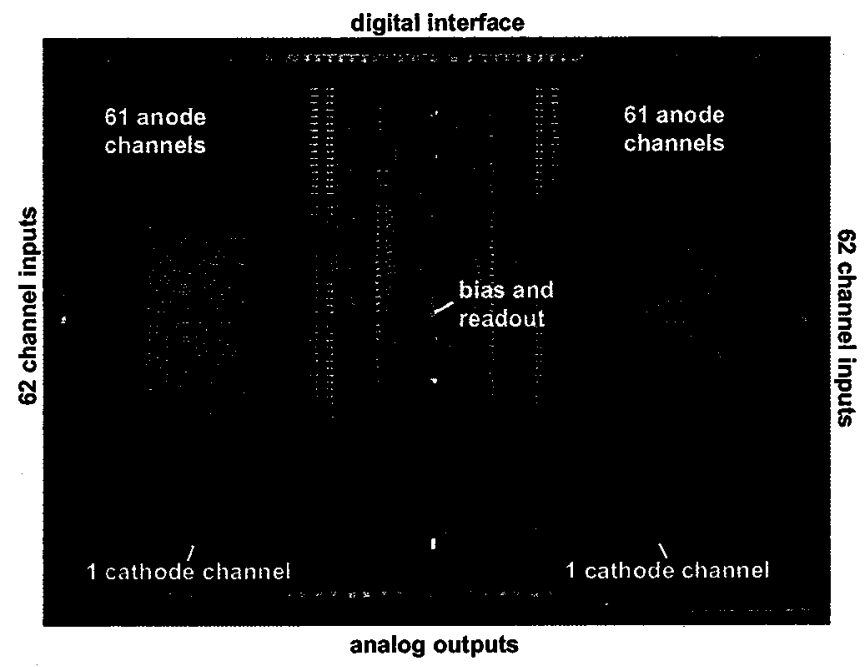

Fig. 9 - Die photo, layout size $11.3 \mathrm{~mm} \times 8.2 \mathrm{~mm}$.

The ASIC was assembled as shown in Fig. 1(b) and Fig. 1(c), and was characterized without a sensor and, preliminarily, with a 121-pixel CZT sensor of $20 \times 20 \times 5 \mathrm{~mm}^{3}$. Fig. 10 plots the response of the anode channel to a $70 \mathrm{fC}$ input charge for four peaking -time values. The baseline is about $245 \mathrm{mV}$.

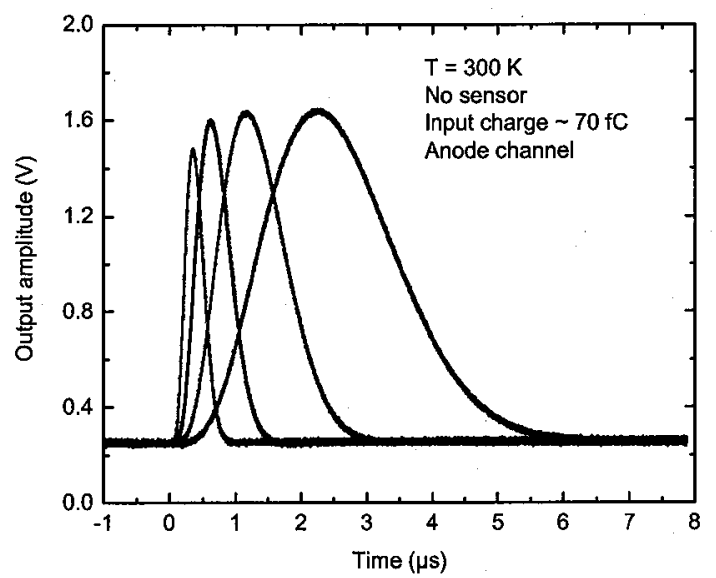

Fig. 10 - Measured response to a $70 \mathrm{fC}$ charge for the four peaking time values.
Fig. 11 shows the ENC measured on cathode and anode channels as function of the peaking time, including the cases without a sensor, with the sensor unbiased and biased. The measurements were taken with a true rms voltmeter at the channel analog output, available through the monitor function. Spectral measurements with a test pulse and no sensor show an increase in the ENC of about $15-20 \%$, due to the noise from the peak detector circuit. In a revised version, we will consider reducing this contribution. This increase becomes negligible with the sensor connected.

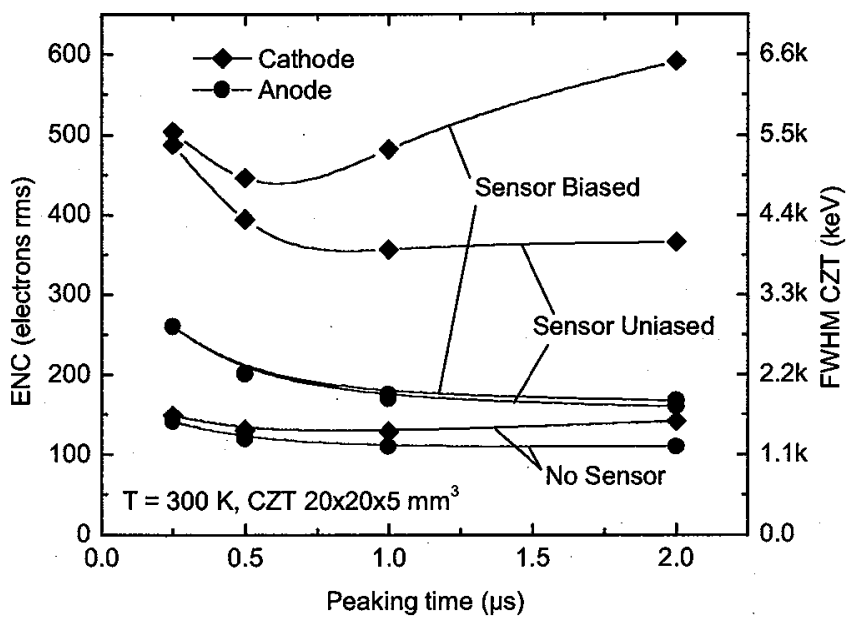

Fig. 11 - Measured ENC versus peaking time.

An integral linearity error below $+/-0.3 \%$ was measured for output signal amplitudes up to $2.25 \mathrm{~V}$ (including the $\approx 250 \mathrm{mV}$ baseline), which corresponds to an input charge of $100 \mathrm{fC}$. A channel-to-channel dispersion of $5.2 \mathrm{mV} \mathrm{rms}$ and $1.5 \% \mathrm{rms}(2$ $\%$ rms at $0.25 \mu$ s peaking time) were, respectively, measured on the baseline and gain.

The timing response of the anode channels was characterized, with the test pulse, in terms of resolution and time walk. Fig. 12(a) shows the measured resolution as function of the injected charge for the four peaking times. Values below $700 \mathrm{ps}$ were measured at a peaking time of $250 \mathrm{~ns}$ for charges above 30 $\mathrm{fC}$, limited by the noise from the TAC and downstream stages. For low amplitudes, the resolution was considerably worse than the theoretical value (1). Further analysis revealed that the increase is dominated by the noise from the shaper and peak detector. For example, we observed that the resolution increases faster than the increase in peaking time (see Section III). Further confirmation comes from the additional measurements (open symbols) shown in Fig.12(a), obtained by loading the input of the channel with a $3 \mathrm{pF}$ capacitor. Preceding a factorof-two increase in rms noise at the shaper's output, only a negligible increase is observed in timing resolution (compare the solid symbols to the open symbols), especially at longer peaking times. We clearly overlooked the impact of the noise from the shaper and peak detector on the timing resolution; it will be resolved in a later version. 

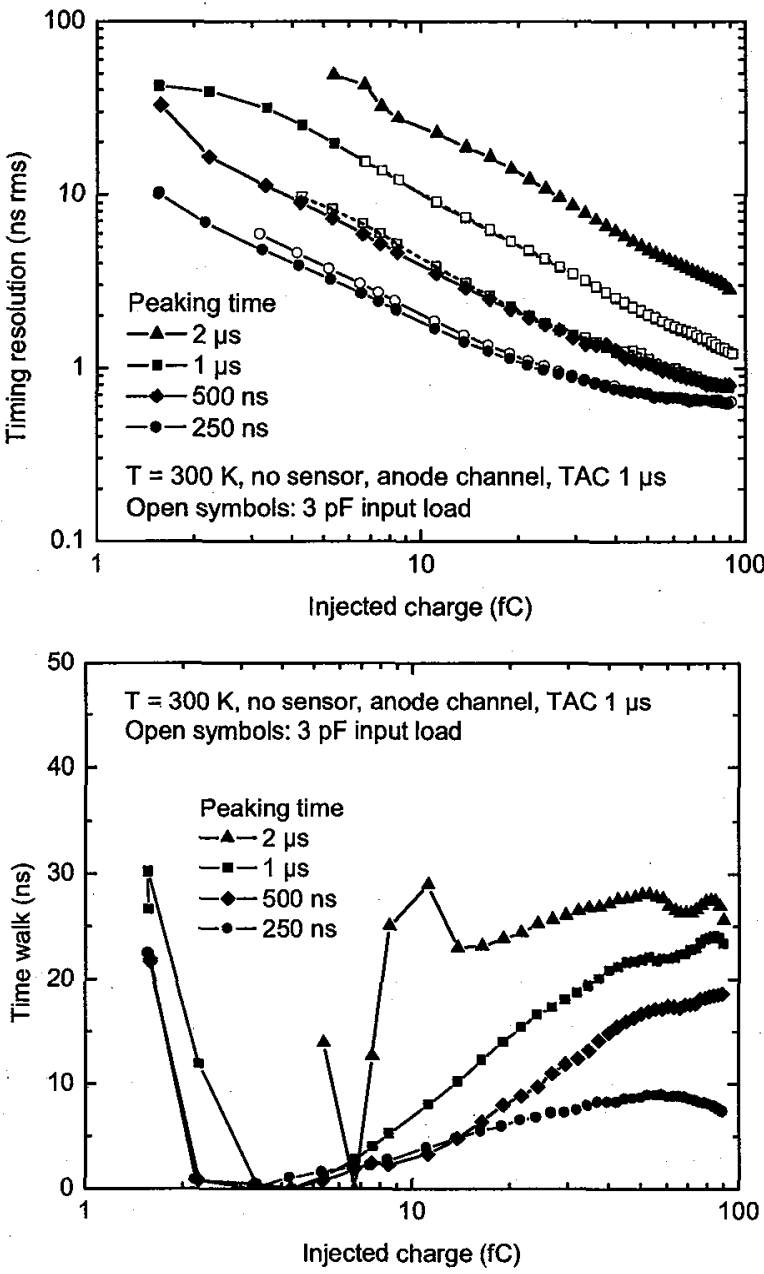

(a)

Fig. 12 - Measured anode- channel timing resolution (a) and time walk (b).

Fig. 12(b) shows the measured time walk, referenced to the minimum value, as function of the injected charge for the four peaking times. The time walk is contained within $8 \mathrm{~ns}$ and 30 ns, respectively, at the shortest and longest peaking time. Almost identical results were obtained with the $3 \mathrm{pF}$ capacitor at the input.

The timing response of the cathode channel was characterized using the three implemented methods: preamplifier multithreshold, unipolar threshold, and bipolar peaking time. It was accomplished via injecting charge with the integrated test pulse and test ramp generators. The duration of the ramp was set to $500 \mathrm{~ns}$ and the injected charge was altered by changing the ramp's slope. The peaking time in the energy section of the cathode channel was set to $250 \mathrm{~ns}$. The results from this characterization are reported here, while a comparative analysis will be published later.

For the preamplifier multi-threshold, Fig. 13(a) shows the measured resolution as function of the injected charge for the four peaking times. The solid symbols correspond to the $500 \mathrm{~ns}$ test ramp, while the open symbols represent the test pulse, for which only the first threshold was characterized.

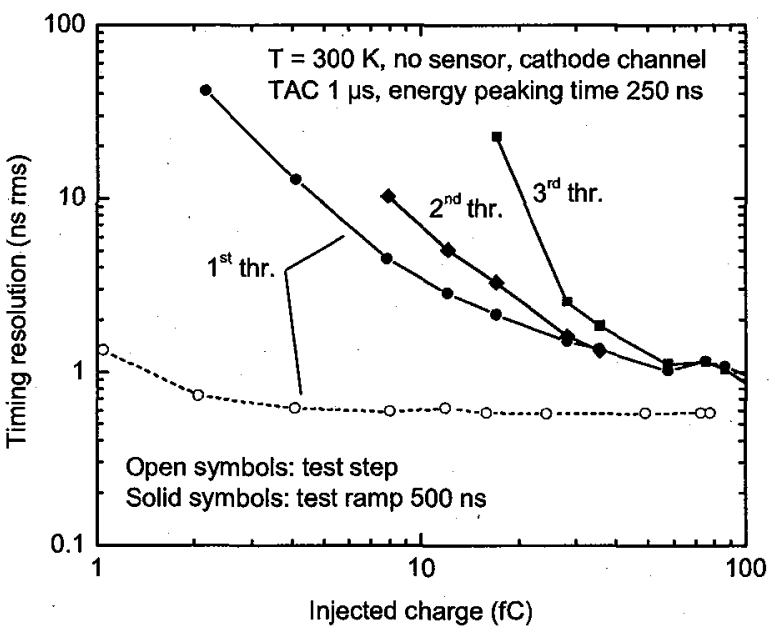

(a)

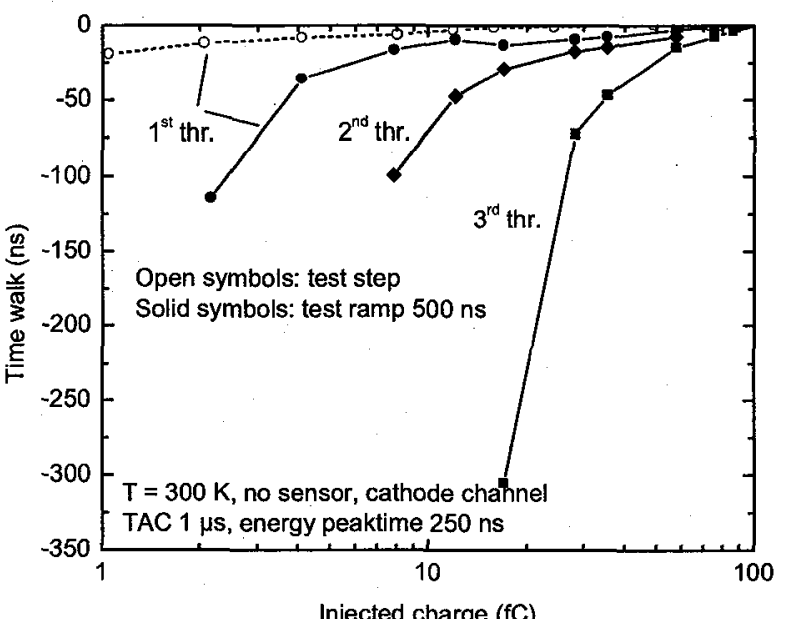

(b)

Fig. 13 - Measured cathode timing resolution (a) and time walk (b) using the preamplifier multi-threshold method.

The first threshold was set at about $0.5 \mathrm{fC}$, while the second and third thresholds were set to cover as much as possible the range of interest from $1 \mathrm{fC}$ to $100 \mathrm{fC}$. We noted that, due to the poor signal level, a minimum detectable charge of $2 \mathrm{fC}, 8 \mathrm{fC}$, and $17 \mathrm{fC}$ for the first, second, and third threshold, respectively. were measured with the ramp. Excellent timing resolution was measured with the test pulse, while, in the most realistic case of the ramp, the timing resolution was below $20 \mathrm{~ns}$ for charges above $3 \mathrm{fC}$. Fig. 13(b) shows the corresponding time walk referenced to the maximum value, where, for the first threshold, values in excess of $100 \mathrm{~ns}$ were obtained for the test ramp. The difference in timing between the thresholds, properly calibrated, provides a measure of the slope of the test ramp that allows for timewalk correction. To have a difference available, at least two thresholds must be crossed, which in Fig. 14 means having a charge above $8 \mathrm{fC}$. For signals from the sensor, this also constitutes a direct measurement of the ionized charge (i.e., the energy). Suitable calibration can also provide the corrective coefficient for the time walk associated with the first threshold.

For a unipolar threshold set at about $0.5 \mathrm{fC}$, Fig. 14(a) shows the measured resolution as function of the injected charge for the four peaking times. The solid symbols correspond to the $500 \mathrm{~ns}$ test ramp, and the open symbols to the test pulse. In all 
cases, we measured a timing resolution below $20 \mathrm{~ns}$. For the ramp, the impact of the signal's reduced speed on the resolution is apparent. Fig. 14(b) shows the corresponding time walk referenced to the maximum value. Especially in the case of the ramp, the time walk can be as high as $400 \mathrm{~ns}$ for low charges.

For the bipolar peaking time, Fig. 15(a) and Fig. 15(b) show the measured resolution of the test ramp as function of the injected charge for the four peaking times. . Due to a design error, for a given charge the pulse amplitude was proportional to the peaking time. This reduction in gain strongly affected the performance at low peaking times and will be corrected in a revision. Additionally, we saw considerable pick-up from the digital signals that prevented the characterization for charges below $4 \mathrm{fC}$. Finally, as in the case of the anode, the noise from the shaper and peak detector strongly affected these measurements, issues that also will be resolved later. .

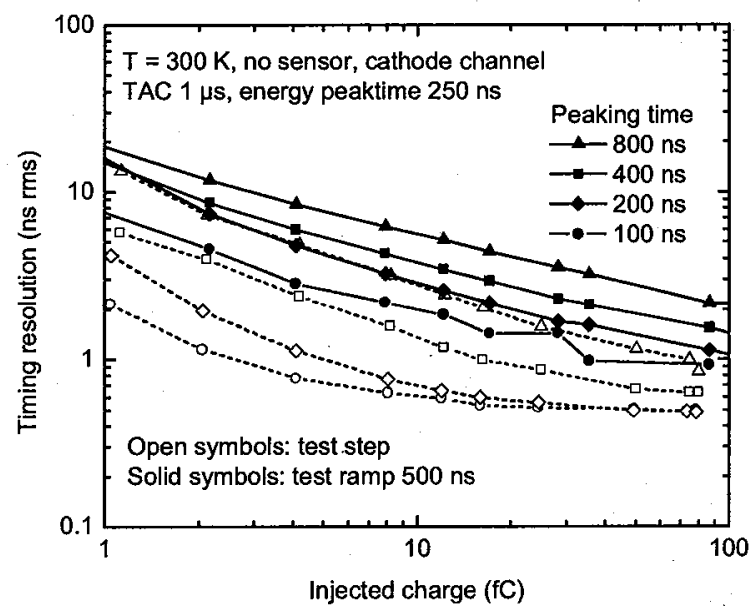

(a)

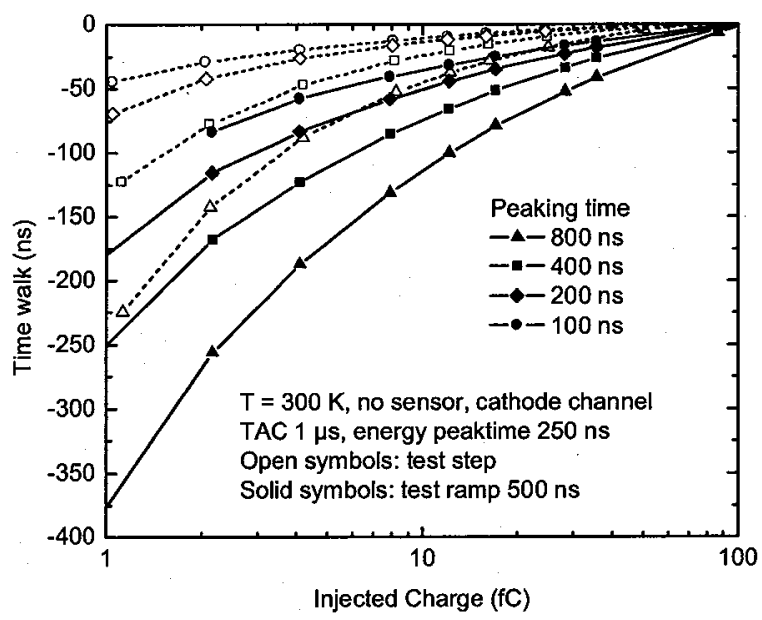

(b)

Fig. 14 - Measured cathode timing resolution (a), and time walk (b), using the unipolar method.

As discussed in Section II, the amplitude of the pulse from the peak detector, being proportional to the slope, is a measure of the energy. A resolution between 300- and 500-rms electrons was detected in the range of the four peaking times. Fig. 15(c) shows the measured peak amplitude on the positive- and negative-peaks as function of the injected charge (i.e., as a function of the slope).

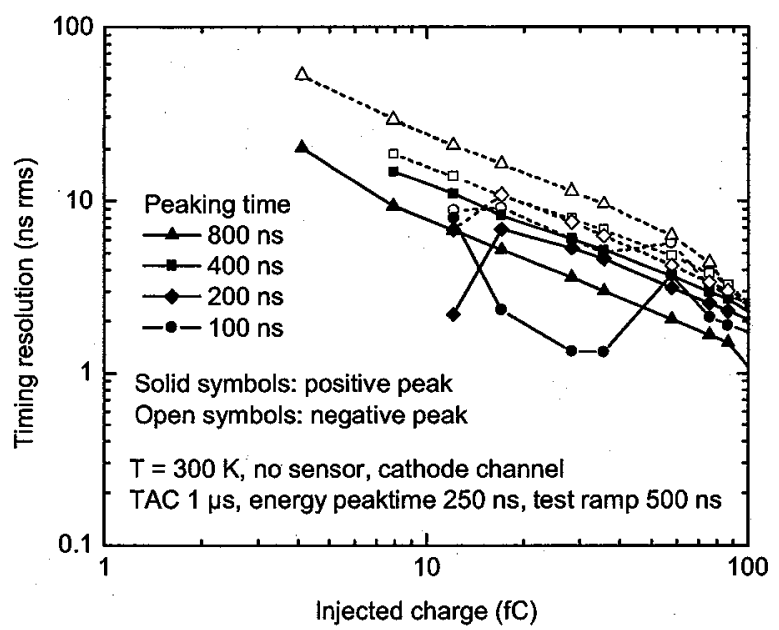

(a)

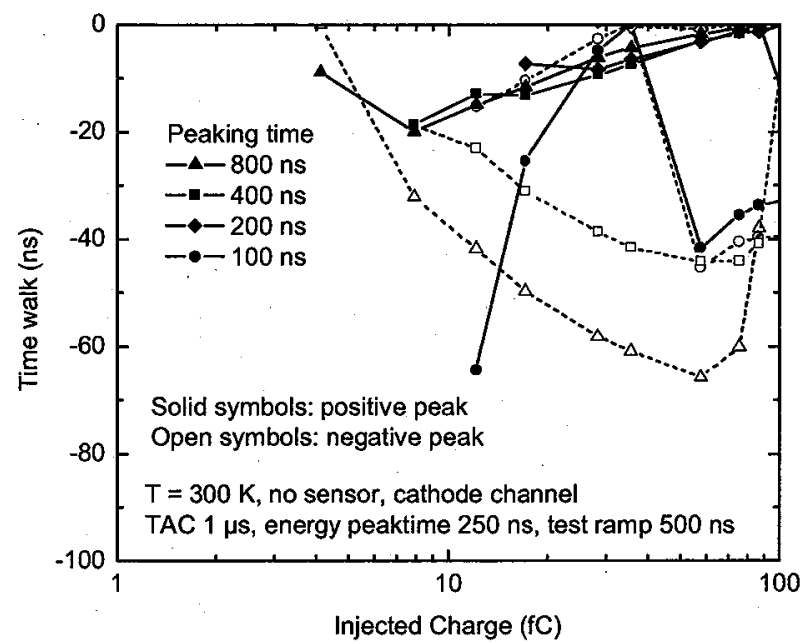

(b)

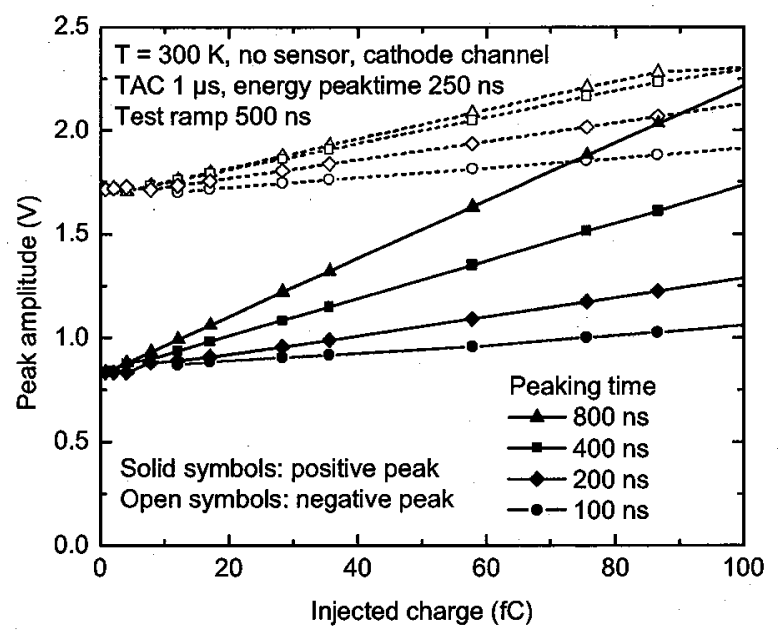

(c)

Fig. 15 - Measured cathode timing resolution (a), time walk (b), and peak voltage (c) using the bipolar peaking time method.

Fig. 16(a) plots a single pixel spectrum from a ${ }^{137} \mathrm{Cs}$ source acquired at a peaking time of $1 \mu \mathrm{s}$. Fig. 16(b) shows the corrected spectrum using the cathode/anode ratio. The resolution is about $4.8 \mathrm{keV}(0.72 \%)$ at $662 \mathrm{keV}$, while the contribution from the electronics and leakage current is on the order of $2.1 \mathrm{keV}$. 

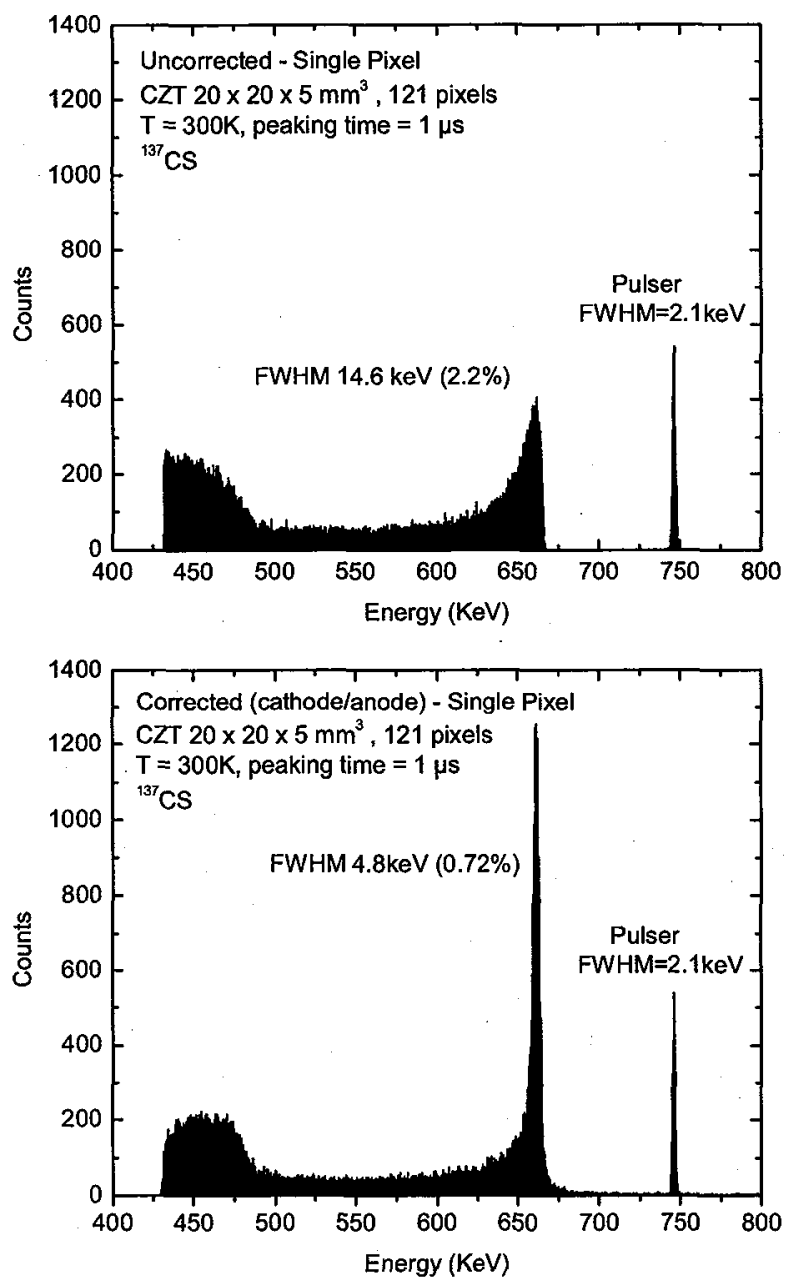

(a)

Fig. 16 - Single pixel spectrum from a ${ }^{137} \mathrm{Cs}$ source: (a) uncorrected, and (b) corrected using the cathode/anode ratio.

\section{CONCLUSIONS AND FUTURE WORK}

We demonstrated that the performance of our first prototype of an ASIC for 3D position-sensitive pixelated CZT sensors shows good agreement with the design in its functionality and energy resolution, where less than 200 electrons rms are measured with the sensor connected and biased. Margins for improvement were identified in the anode timing, where a final resolution below $20 \mathrm{~ns}$ can reasonably be expected from a revision. Three different methods were characterized for resolving cathode timing that will undergo further comparative analyses. . Digital pick-up and low gain affect bipolar timing and will be resolved in a revision. Calibration routines also will be implemented using the integrated test pulse and test ramp. Finally, we will undertake extensive characterization with pixelated CZT sensors.

\section{ACKNOWLEDGMENTS}

The authors are grateful to Fred Ferraro, from eV Products, Div. of II-VI Inc., and Dr. Alan Janos, from DNDO-DHS, for their encouragement and support. A special acknowledgment goes to Avril D. Woodhead for assistance with editing. This project was founded by the Domestic Nuclear Detection Office (DNDO) of the US Department of Homeland Security.

This manuscript has been authored by employees of Brookhaven Science Associates, LLC under Contract No. DE-AC0298CH10886 with the U.S. Department of Energy. The publisher by accepting the manuscript for publication acknowledges that the United States Government retains a non-exclusive, paid-up, irrevocable, world-wide license to publish or reproduce the published form of this manuscript, or allow others to do so, for United States Government purposes.

\section{REFERENCES}

[1] A. Owens and A. Peacock, "Compound semiconductor radiation detectors", Nucl. Instrum. Methods, A531, pp. 18-37, 2004.

[2] C. H. Malden and R. D. Speller, "A CdZnTe array for the detection of explosives in baggage by energy-dispersive $\mathrm{X}$-ray diffraction signatures at multiple scatter angles", Nucl. Instrum. Meth., A449, pp. 408-415, 2000.

[3] R. Arlt, J. Brutscher, R. Gunnink, V. Ivanov, K. Parnham, S. A. Soldner, and $\mathbf{J}$. Stein, "Use of $\mathrm{CdZnTe}$ detectors in hand-held and portable isotope identifiers to detect illicit trafficking of nuclear material and radioactive sources", IEEE NSS 2000 Conf. Record, pp. 4/18-4/23, 2001.

[4] T. H. Prettyman, K. D. Ianakiev, C. E. Moss, S. A. Soldner, M. R. Sweet, and M. C. Browne, "Developments of high efficiency, multi-element $\mathrm{CdZnTe}$ detectors for portable measurement applications", Journal of Radioanalytical and Nuclear Chemistry, vol. 248, pp. 295-300, May 2001.

[5] P. N. Luke, M. Amman, J. S. Lee, B. A. Ludewigt, and H. Yaver, "CdZnTe coplanar grid detector array for environmental remediation", Nucl. Instrum. Meth., A458, pp. 319-324, 2001.

[6] T. H. Prettyman, W. C. Feldman, K. R. Fuller, S. A. Storms, S. A. Soldner, C. Szeles, F. P. Ameduri, D. J. Lawrence, M. C. Browne, and C. E. Moss, "CdZnTe Gamma-ray spectrometer for orbital planetary missions", IEEE Trans. Nucl. Sci., vol. 49, pp. 1881-1886, 2002.

[7] O. Limousin, "New trends in CdTe and CdZnTe detectors for X- and gamma-ray applications", Nucl. Instrum. Meth., A504, pp. 24-37, 2003.

[8] W. Li, S. Li, S. Zhu, S. Yin, B. Zhao, G. Chen, S. Yin, H. Yuan, and H. Xu, "Mercuric iodide single crystals for nuclear radiation detectors", IEEE Trans. Nucl. Sci., vol. 43, pp. 1369-1371, 1996.

[9] L. J. Meng, Z. He, B. Alexander, and J. Sandoval, "Spectroscopic performance of thick HgI2 detectors", IEEE Trans. Nucl. Sci., vol. 53, pp. 1706-1712, 2006.

[10] Z. He and R. D. Vigil, "Investigation of pixelated HgI2 Gamma-ray spectrometers", Nucl. Instrum. Meth., A492, pp. 387-401, 2002.

[11] R. Redus, M. Squillante, and J. Lund, "Electronics for high resolution spectroscopy with compound semiconductors", Nucl. Instrum. Meth., A380, pp. 312-317, 1996.

[12] J. C. Lund, J. M. VanScyoc III, R. B. James, D. S. McGregor, and R. W. Olsen, "Large volume room temperature gamma-ray spectrometers from CdZnTe", Nucl. Instrum. Meth., A380, pp. 256-261, 1996.

[12] R. Redus, M. Squillante, and J. Lund, "Electronics for high resolution spectroscopy with compound semiconductors", Nucl. Instrum. Meth., A380, pp. 312-317, 1996.

[13] M. Amman and P. Luke, "Optimization criteria for coplanar-grid detectors”, IEEE Trans. Nucl. Sci., vol. 46, pp. 205-212, 1999.

[14] A. Burger, K. Chattopadhyay, H. Chen, X. Ma, J. O. Ndap, M. Schieber, T. E. Schlesinger, H. W. Yao, J. Erickson, and R. B. James, "Defects, in CZT crystals and their relationship to gamma-ray detector performance", Nucl. Instrum. Meth., A448, pp. 586-590, 2000.

[15] F. Mathy, A. Gliere, E. G. d'Aillon, P. Masse, M. Picone, J. Tabary, and L. Verger, "A three-dimensional model of CdZnTe gamma-ray detector and its experimental validation", IEEE Trans. Nucl. Sci., vol. 51, pp. 2419-2426, 2004. [16] E. G. d'Aillon, M. C. Gentet, G. Montemont, J. Rustique, and L. Verger, "Simulation and experimental results on monolithic CdZnTe gamma-ray detectors", IEEE Trans. Nucl. Sci., vol. 52, pp. 3096-3102, 2004.

[17] A. Shor, Y. Eisen, and I. Mardor, "Gamma spectroscopy with pixelated CdZnTe detectors”, IEEE Trans. Nucl. Sci, vol. 51, pp. 1204-1208, 2004.

[18] A. E. Bolotnikov, G. C. Camarda, G. W. Wright, and R. B. James, "Factors limiting the performance of CdZnTe detectors", IEEE Trans. Nucl. Sci., vol, 52, pp. 589-598, 2005.

[19] D. S. Bale and C. Szeles, "Design of high-performance CdZnTe quasihemispherical Gamma-ray CAPture plus detectors", Proc. of SPIE, vol. 6319, 2006. 
[20] Z. He, W. Li, G. F. Knoll, D. K. Wehe, J. Berry, and C. M. Stahle, "3-D position sensitive CdZnTe gamma-ray spectrometers", Nucl. Instrum. Methods, A422, pp. 173-178, 1999.

[21] W. Li, Z. He, G. F. Knoll, D. K. Wehe, and Y. F. Du, "A modeling method to calibrate the interaction depth in 3-D position sensitive CdZnTe gamma-ray spectrometers", IEEE Trans. Nucl. Sci., vol. 47, pp. 890-894, 2000.

[22] F. Zhang, Z. He, and D. Xu, "Improved Resolution for 3-D Position Sensitive CdZnTe Spectrometers", IEEE Trans. Nucl. Sci., vol. 51, pp. 24272431, 2004.

[23] G. De Geronimo, P. O'Connor, and J. Grosholz; "A generation of CMOS readout ASICs for CZT detectors", IEEE Trans. Nucl. Sci., vol. 47, pp. 1857 $1867,2000$.

[24] G. De Geronimo and P. O'Connor, "MOSFET optimization in deep submicron technology for charge amplifiers", IEEE Trans. Nucl. Sci., vol. 52 , pp. 3223-3232, 2005.

[25] G. De Geronimo, J. Fried, E. Frost, B. F. Phlips, E. Vernon, and E. A Wulf, "Front-end ASIC for a Silicon Compton telescope", Proc. 2007 IEEE Nucl. Sci. Symp., Hawii, Oct. 2007.

[26] G. De Geronimo, P. O'Connor, and J. Grosholz, "A CMOS baseline holder (BLH) for readout ASICs", IEEE Trans. Nucl. Sci., vol. 47, pp. 818-822, Jun. 2000 .
[27] G. De Geronimo, P. O'Connor, and A. Kandasamy, "Analog CMOS peak detect and hold circuits, Parts 1 and 2", Nucl. Instrum. Methods, A484, pp. 533$556,2002$.

[28] A. Dragone, G. De Geronimo, J. Fried, A. Kandasamy, P. O'Connor, and E. Vernon, "The PDD ASIC: Highly Efficient Energy and Timing Extraction for High-Rate Applications", 2005 IEEE Nucl. Sci. Symp. Conf. Rec., vol. 2, pp. 914-918, 2006.

[29] U. Lachish, "Driving spectral resolution to the noise limit in semiconductor gamma detector arrays", IEEE Trans. Nucl. Sci., vol. 48, pp. 520-523, Jun. 2001.

[30] E. G. d'Aillon, M. C. Gentet, G. Montemont, J. Rustique, and L. Verger, "Simulation and experimental results on monolithic CdZnTe gamma-ray detectors", IEEE Trans. Nucl. Sci., vol. 52, pp. 3096-3102, Dec. 2005.

[31] G. De Geronimo, "Low-noise electronics for radiation sensors" in Circuits for Emerging Technologies, K. Iniewski editor, to be published.

[32] A. R. Frolov, T. V. Oslopova, and Y. N. Pestov, "Double threshold discriminator for timing measurements", Nucl. Instrum. Meth., A356, pp. 447$451,1995$. 